\title{
Children's Exposure to emerging Bisphenols other than Bisphenol A: It's time for more biomonitoring studies
}

\author{
Fiorella Lucarini ${ }^{1}$ and Davide Staedler ${ }^{1,2 *}$ \\ ${ }^{1}$ Department of Biomedical Sciences, University of Lausanne, Switzerland \\ ${ }^{2}$ Scitec Research SA, Switzerland
}

*Corresponding author: Lucarini F, Staedler D, Department of Biomedical Sciences, University of Lausanne, 1011 Lausanne, Switzerland, Scitec Research SA, Av. De Provence 18, 1007 Lausanne, Switzerland.

To Cite This Article: Fiorella L, Davide S. Children's Exposure to emerging Bisphenols other than Bisphenol A: It's time for more biomonitoring studies. 2020 - 9(6). AJBSR.MS.ID.001445. DOI: 10.34297/AJBSR.2020.09.001445.

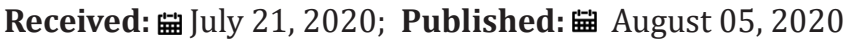

\section{Opinion}

There is a great deal of evidence which has raised the need for various governmental organizations to regulate the use of bisphenol A (BPA) as plasticizer [1-4]. Indeed, BPA has shown endocrine disruptor effects, associated with a wide range of health outcomes [5,6]. Furthermore, biomonitoring investigations have frequently detected the presence of BPA in human urine, serum and breast milk; therefore, the exposure to this substance has been reported to be widespread [7-9].

Because of the endocrine disrupting potency, BPA exposure to young children is particularly harmful and for this reason the use of BPA in baby bottles and pacifiers has been banned in several countries such as United States, Canada, and Europe [10]. In addition, in 2017, BPA has been added to the list of substances of very high concern by the European Chemical Agency (ECHA) [11]. Following these actions, other bisphenols (BPs) have recently emerged as alternative to bisphenol $\mathrm{A}$ in the production of many consumer products. Many of these bisphenols have been identified in environmental studies, such as bisphenol AF (BPAF), bisphenol AP (BPAP), bisphenol B (BPB), bisphenol BP (BPBP), bisphenol C (BPC), bisphenol E (BPE), bisphenol F (BPF), bisphenol G (BPG), bisphenol M (BPM), bisphenol P (BPP), bisphenol PH (BPPH), bisphenol S (BPS), bisphenol TMC (BPTMC) and bisphenol Z (BPZ) [12]. Given the structural similarity between these BP analogues and bisphenol A, it is highly likely that these BPs have similar biological activity as bisphenol A also with regard to health effects $[13,14]$. Indeed, some toxicological studies on these BPs, such as bisphenol AF, bisphenol B, bisphenol C, bisphenol E, bisphenol
$\mathrm{F}$, and bisphenol S, have shown endocrine disruption activities comparable or even greater than that of BPA [15-19]. Very few biomonitoring studies have investigated the human exposure to these bisphenol analogues [20]. In particular, considering urine samples as the best approach to monitor total BPs concentrations, only nine BPs (i.e. BPA, BPAF, BPAP, BPB, BPF, BPM, BPP, BPS, BPZ) between the most common bisphenols, have been researched in human urinary samples [12]. Among these studies, BPA, BPF and BPS resulted the most frequently detected in adults and children with BPA generally detected in $74-100 \%$ of the samples [21].

The simultaneous investigation of the most common BPs (i.e. the 14 BPs listed above) has been reported for the first time in a very recent study. This work has analyzed the presence of these BP analogues in the urine extracted from disposable diapers in young Swiss children aged between 6 and 36 months of life $(n=109)$ [22].

This study has shown a decrease in the detection frequency of urinary BPA compared to previous studies and an increased detection frequency of bisphenol $\mathrm{M}$ and bisphenol C. In particular, BPM and BPC were the most frequently detected BP analogues with a detection frequency of $25 \%$ and $23 \%$, respectively. Meanwhile, BPA was detected only in $7 \%$ of the samples.

This change in detection frequency is probably since BPA is increasingly being replaced by its BP analogues. Indeed, more and more often different kinds of children's consumer products are claimed BPA free. On the other hand, there is no information concerning the extent to which the other BPs are actually used nor is there any legal obligation for producers of chemicals of release 
this information. Furthermore, in the same study, significantly higher concentrations of BPM were detected in infants with respect to toddlers. Although biomonitoring studies suffer from the disadvantage of not being able to determine the routes of exposure, this result highlights different sources of exposure for infants and toddlers that should be further characterized.

All these findings highlight the need for extended biomonitoring studies on BP analogues exposure including larger population size. Biomonitoring is an essential tool to supervise human exposure to emerging chemical compounds [23], and the most at-risk classes such as children. Therefore, the still scarce investigations on these BP derivatives, should be enlarged to obtain statistically significative exposure data, useful as tools for the implementation of human health protection measures as has been done for bisphenol $\mathrm{A}$ in the past.

In conclusion, these studies should be taken as starting point for assessing human exposure to potentially harmful substances and more biomonitoring data should be carried out before embarking on new routes that could be even less safe than BPA.

\section{Reference}

1. (2008) NTP, (US National Toxycology Program). In CERHR (Ed) NTP-CERHR Monograph on the Potential Human Reproductive and Developmental Effects of Bisphenol A: 1-324.

2. Seachrist DD, Bonk KW, Ho SM, Prins GS, Soto AM, et al. (2016) A review of the carcinogenic potential of bisphenol A. Reprod Toxicol 59: 167-182.

3. ECHA, (European Chemicals Agency) (2015) Opinion on an Annex XV Dossier Proposing Restrictions on Bisphenol A. Compiled Version Prepared by the ECHA Secretariat of RAC's Opinion (Adopted 5 June 2015) and SEAC's Opinion (Adopted 4 December 2015).

4. Ma Y, Liu H, Wu J, Yuan L, Wang Y, et al. (2019) The adverse health effects of bisphenol A and related toxicity mechanisms. Environ Res 176: 108575.

5. Beausoleil C, Emond C, Cravedi J P, Antignac J P, Applanat M, et al. (2018) Regulatory identification of BPA as an endocrine disruptor: Context and methodology. Mol Cell Endocrinol 475: 4-9.

6. Ejaredar M, Lee Y, Roberts DJ, Sauve R, and Dewey D (2017) Bisphenol A exposure and children's behavior: A systematic review. J Expo Sci Environ Epidemiol 27(2): 175-183.

7. Deceuninck Y, Bichon E, Marchand P, Boquien CY, Legrand A, et al. (2015) Determination of bisphenol A and related substitutes/analogues in human breast milk using gas chromatography-tandem mass spectrometry. Anal Bioanal Chem 407(9): 2485-97.

8. Huang RP, Liu ZH, Yin H, Dang Z, Wu PX, et al. (2018) Bisphenol A concentrations in human urine, human intakes across six continents, and annual trends of average intakes in adult and child populations worldwide: A thorough literature review. Sci. Total Environ. 626: 971981.
9. Owczarek K, Kubica P, Kudlak B, Rutkowska A, Konieczna A, et al. (2018) Determination of trace levels of eleven bisphenol A analogues in human blood serum by high performance liquid chromatography-tandem mass spectrometry. Sci Total Environ 626: 1362-1368.

10. (2011) EU, (European Commission) Bisphenol A: EU ban on baby bottles to enter into force tomorrow.

11. (2017) ANSES, (French Agency for Food, Environmental and Occupational Health and Safety) Annex XV report. Proposal for identification of a substance of very high concern based on the criteria set out in REACH Article 57. Substance Name(s): 4,4'-isopropylidenediphenol (Bisphenol A). EC Number: 201-245-8. CAS Number: 80-05-7.

12. Chen D, Kannan K, Tan H, Zheng Z, Feng Y L, et al. (2016) Bisphenol Analogues Other Than BPA: Environmental Occurrence, Human Exposure, and Toxicity-A Review. Environ Sci Technol 50(11): 54385453.

13. ANSES (2013) Reprotoxic substances and endocrine disruptors. Compounds of the bisphenol family: bisphenols M, S, B, AP, AF, F and BADGE. Expert Committee Report.

14. Rosenmai AK, Dybdahl M, Pedersen M, Alice van Vugt-Lussenburg BM, Wedebye E B, et al. (2014) Are structural analogues to bisphenol a safe alternative? Toxicol Sci 139(1): 35-47.

15. Pelch K, Wignall JA, Goldstone AE, Ross PK, Blain RB, et al. (2019) A scoping review of the health and toxicological activity of bisphenol A (BPA) structural analogues and functional alternatives. Toxicology 424: 152235 .

16. Macczak A, Cyrkler M, Bukowska B, Michalowicz J (2017) Bisphenol A, bisphenol $\mathrm{S}$, bisphenol $\mathrm{F}$ and bisphenol $\mathrm{AF}$ induce different oxidative stress and damage in human red blood cells (in vitro study). Toxicol In Vitro 41: 143-149.

17. Serra H, Beausoleil C, Habert R, Minier C, Picard-Hagen N, et al. (2019) Evidence for Bisphenol B Endocrine Properties: Scientific and Regulatory Perspectives. Environ Health Perspect 127(10): 106001.

18. Ijaz S, Ullah A, Shaheen G, Jahan S (2020) Exposure of BPA and its alternatives like BPB, BPF, and BPS impair subsequent reproductive potentials in adult female Sprague Dawley rats. Toxicol Mech Methods 30(1): 60-72.

19. Goldinger DM, Demierre AL, Zoller O, Rupp H, Reinhard H, et al. (2015) Endocrine activity of alternatives to BPA found in thermal paper in Switzerland. Regul Toxicol Pharmacol 71(3): 453-62.

20. Thayer KA, Pelch KE, Birnbaum LS, Bucher JR (2016) Bisphenols: More unnecessary surprises. Endocrine Disruptors 4(1): e1131032.

21. Lehmler H J, Liu B, Gadogbe M, Bao W (2018) Exposure to Bisphenol A Bisphenol F, and Bisphenol S in U.S. Adults and Children: The National Health and Nutrition Examination Survey 2013-2014. ACS Omega 3(6): 6523-6532.

22. Lucarini F, Krasniqi T, Bailat Rosset G, Roth N, Hopf N B, et al. (2020) Exposure to New Emerging Bisphenols Among Young Children in Switzerland. Int J Environ Res Public Health 17(13): 4793.

23. Louro H, Heinala M, Bessems J, Buekers J, Vermeire T, et al. (2019) Human biomonitoring in health risk assessment in Europe: Current practices and recommendations for the future. Int J Hyg Environ Health 222(5): 727-737. 